\title{
Connecting the Stimuli-Responsive Rheology of Biopolymer Hydrogels to Underlying Hydrogen-Bonding Interactions
}

\author{
Giulia Giubertoni, Federica Burla, Huib J. Bakker,* and Gijsje H. Koenderink*
}

Cite This: Macromolecules 2020, 53, 10503-10513

Read Online

ABSTRACT: Many biopolymer hydrogels are environmentally responsive because they are held together by physical associations that depend on $\mathrm{pH}$ and temperature. Here, we investigate how the $\mathrm{pH}$ and temperature responses of the rheology of hyaluronan hydrogels are connected to the underlying molecular interactions. Hyaluronan is an essential structural biopolymer in the human body with many applications in biomedicine. Using two-dimensional infrared spectroscopy, we show that hyaluronan chains become connected by hydrogen bonds when the $\mathrm{pH}$ is changed from 7.0 to 2.5 and that the bond density at $\mathrm{pH} 2.5$ is independent of temperature. Temperature-dependent rheology measurements

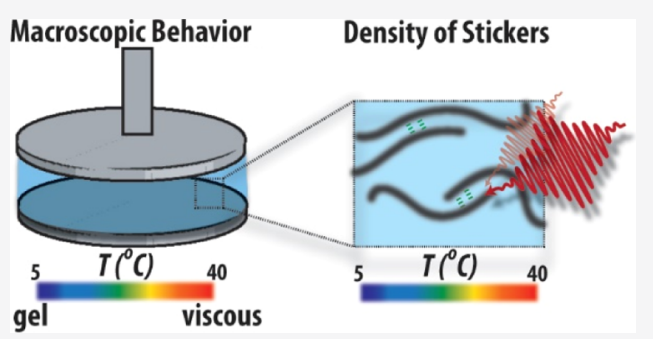
show that because of this hydrogen bonding the stress relaxation at $\mathrm{pH} 2.5$ is strongly slowed down in comparison to $\mathrm{pH} 7.0$, consistent with the sticky reptation model of associative polymers. From the flow activation energy, we conclude that each polymer is cross-linked by multiple (5-15) hydrogen bonds to others, causing slow macroscopic stress relaxation, despite the short time scale of breaking and reformation of each individual hydrogen bond. Our findings can aid the design of stimuli-responsive hydrogels with tailored viscoelastic properties for biomedical applications.

\section{INTRODUCTION}

Hydrogels are an appealing class of polymer materials because of their responsiveness to environmental stimuli such as temperature, $\mathrm{pH}$, and ionic strength. ${ }^{1}$ Hydrogels derive this responsiveness from the presence of reversible associations between the constituent polymer chains, such as electrostatic, hydrogen-bonding, or hydrophobic associations or metal complexation. ${ }^{2}$ Stimuli-responsive hydrogels have a wide range of applications, such as adhesives, ${ }^{3}$ controlled drug delivery, ${ }^{4}$ surrogate extracellular materials for tissue regeneration, ${ }^{5}$ and bioinks for printing of soft tissues. ${ }^{6}$

For biomedical applications, it is advantageous to create hydrogels from biopolymers that are naturally present in the human body, as these can capture both the mechanical and biological properties of the extracellular environment. ${ }^{5} \mathrm{~A}$ particularly interesting biopolymer in this context is hyaluronan because of its ubiquity in human tissues and extracellular fluids such as the synovial fluid in joints and the vitreous humor of the eye. ${ }^{7,8}$ Hyaluronan is a linear polyelectrolyte composed of repeating units of D-glucuronic acid and $\mathrm{N}$-acetylD-glucosamine joined by glycosidic bonds. ${ }^{9,10}$ In healthy tissues, hyaluronan has a very high molecular weight of up to $10 \mathrm{MDa}$, corresponding to chain lengths of tens of micrometers. ${ }^{11}$ Its length combined with its anionic nature enable hyaluronan to form soft hydrated viscoelastic fluids. ${ }^{12-14}$ These properties are at the basis of hyaluronan's roles in the lubrication of joints ${ }^{15}$ and in tuning the transport properties and mechanical response of tissues such as skin, cartilage, and brain. ${ }^{16,17}$ Hyaluronan's mechanical properties together with its biochemical interactions regulate cell-matrix and cell-cell interactions, thus influencing cell-cell communication, ${ }^{18}$ wound healing, ${ }^{19}$ and angiogenesis and development. ${ }^{20}$ Accordingly, abnormalities in the expression level or molecular weight of hyaluronan brought about by aging or genetic disorders contribute to various diseases, such as inflammation, ${ }^{21}$ multiple sclerosis, ${ }^{22}$ cancer ${ }^{23,24}$ and cartilage degeneration. $^{25}$

Because of its central importance to human health and disease, there have been many studies of the material properties of isolated hyaluronan (reviewed in ref 14). Like many other biological polyelectrolytes, hyaluronan is responsive to external stimuli, such as temperature, solution $\mathrm{pH}$, salt concentration, and multivalent counterions. ${ }^{9,26,27}$ Of these triggers, the solution $\mathrm{pH}$ has the most marked effect: in a narrow $\mathrm{pH}$ range centered around $\mathrm{pH} 2.5$, hyaluronan solutions turn into soft solids that are stretchable. ${ }^{28-31}$ This special gel state is sometimes referred to as the putty state. ${ }^{32}$ We recently showed with two-dimensional infrared (2DIR) spectroscopy that this gelation process is caused by the $\mathrm{pH}$ dependent formation of specific interchain hydrogen bonds between the carboxyl and amide groups on adjacent chains (see Figure 1a). ${ }^{33}$

Received: July 27, 2020

Revised: November 4, 2020

Published: November 18, 2020 
(a)

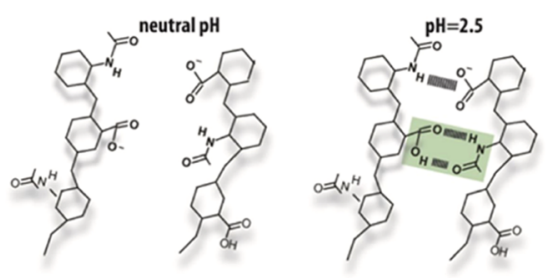

(c)

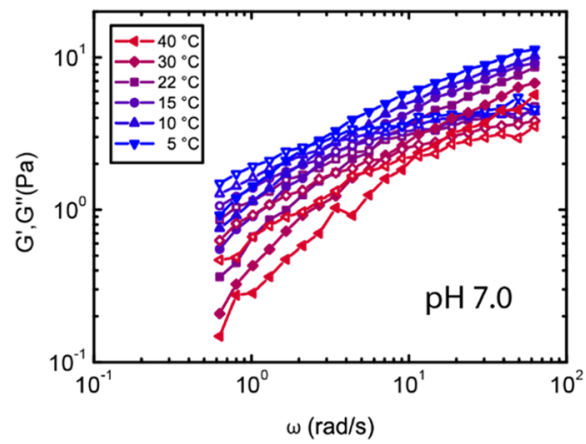

(b)

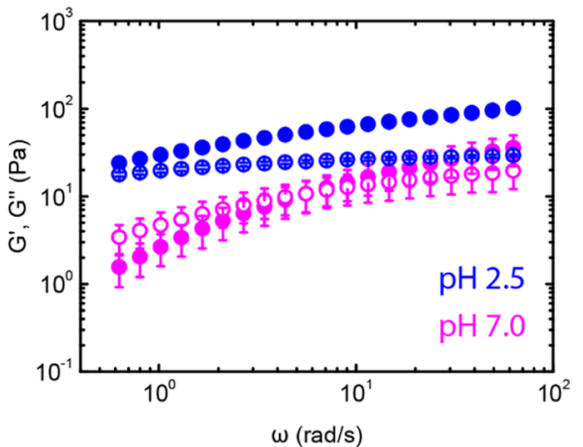

(d)

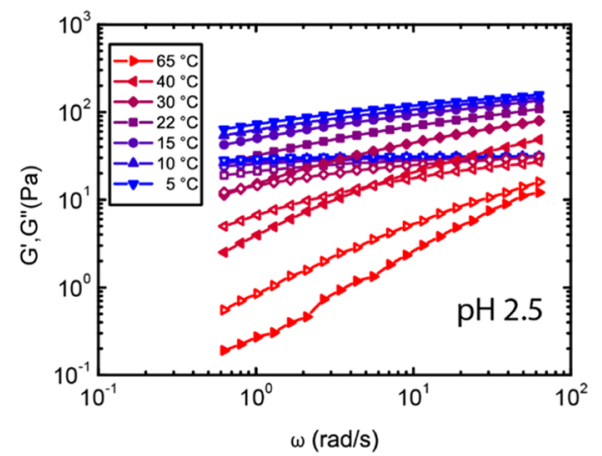

Figure 1. (a) Schematic showing the linear structure of hyaluronan, consisting of repeating disaccharides composed of $N$-acetyl-glucosamine and glucuronic acid. At neutral $\mathrm{pH}(\mathrm{pH} 7)$, the deprotonated carboxyl groups confer a net negative charge. At $\mathrm{pH} 2.5$, the chains associate by the formation of hydrogen bonds between the carboxylic acid, the deprotonated carboxylate anion, and the amide groups of the hyaluronan polymers. ${ }^{39}$ (b) Frequency spectra of the linear viscoelastic shear moduli of hyaluronan hydrogels $(c=10 \mathrm{mg} / \mathrm{mL})$ at room temperature $\left(T=22{ }^{\circ} \mathrm{C}\right)$ at $\mathrm{pH} 7.0$ (pink) and pH 2.5 (blue). Symbols denote the elastic (solid circles) and viscous (open circles) shear moduli. (c) Temperature dependence of the frequency spectra of the shear moduli of hyaluronan solutions at $\mathrm{pH}$ 7.0. (d) Corresponding frequency spectra at $\mathrm{pH}$ 2.5. Note that curves in Figure $1 \mathrm{~b}$ represent an average over three different repeats, while Figure $1 \mathrm{c}, \mathrm{d}$ shows one representative repeat at each temperature.

2DIR spectroscopy is uniquely capable of directly probing molecular interactions by measuring vibrational interactions using an intense femtosecond pump laser to excite the vibrations and a weaker probe beam to measure the induced vibrational responses. We found that at neutral $\mathrm{pH}$ all the carboxyl groups are deprotonated, and thus associative interactions between different polymer chains are prohibited by strong electrostatic repulsion. Hence, at neutral $\mathrm{pH}$, the mechanical properties of hyaluronan solutions are dictated by chain entanglements. By lowering the $\mathrm{pH}$ to 2.5 , close to the isoelectric point of hyaluronan ${ }^{33}$ of $\sim 2.9$, around $80 \%$ of the carboxyl groups are protonated. Protonation weakens the repulsive electrostatic forces between the chains and at the same time provides protonated carboxyl groups that participate in the formation of double hydrogen bonds, together with amide groups and carboxylate anion groups (Figure 1a).

The material properties of responsive hydrogels can be tuned via the lifetime of the reversible cross-links and their number density. There are several theoretical models for associative polymers that predict how the viscoelastic behavior depends on the cross-linker lifetime and density, ${ }^{34,35}$ but it remains challenging to understand the connection between macroscopic and molecular time scales. Hydrogen bonds tend to be short-lived, forming and breaking on a timescale of a few to tens of picoseconds. ${ }^{36}$ Macroscopic stress relaxation in hyaluronan gels at $\mathrm{pH} 2.5$ occurs on much longer time scales, ranging up to minutes. Here, we study the impact of temperature and $\mathrm{pH}$ on the rheology of hyaluronan hydrogels and on the underlying hydrogen-bond-mediated cross-linking by combining rheology and 2DIR spectroscopy. We compare hyaluronan at neutral $\mathrm{pH}$, where the chains interact by excluded volume interactions (i.e., topological entanglements) only, ${ }^{26,37,38}$ and at $\mathrm{pH} 2.5$, where the chains additionally interact by transient hydrogen-bond associations. ${ }^{39}$ We use time-temperature superposition (TTS) rheology to measure the time scale and activation energy for network flow and interpret these findings in the context of the sticky reptation model $^{34,35}$ previously applied to entangled synthetic polymers interacting by thermoreversible cross-linking. ${ }^{40,41}$ We use 2DIR spectroscopy to directly measure the density of interchain hydrogen bonds as a function of temperature.

\section{EXPERIMENTAL SECTION}

Materials and Sample Preparation. Hyaluronan sodium salt in powder form obtained by fermentation of Streptococcus equii with a nominal molecular weight of 1.5-1.8 MDa was obtained from SigmaAldrich. This molecular weight corresponds to a chain length of 3-4 $\mu \mathrm{m}$ and about 3750 disaccharides (considering that the disaccharide units have a molecular weight of $400 \mathrm{Da}$ and a length of $\sim 1 \mathrm{~nm}$ [ref 42]). For rheological measurements, solutions of high molecular weight hyaluronan with concentrations between 5 and $20 \mathrm{mg} / \mathrm{mL}$ were prepared by dissolving accurately weighed amounts of hyaluronic acid in an aqueous solution containing $0.15 \mathrm{M} \mathrm{NaCl}$ (Sigma-Aldrich) and $\mathrm{HCl}$ (Sigma-Aldrich) ranging from 0 to $40 \mathrm{mM}$. The solution $\mathrm{pH}$ was measured with a $\mathrm{pH}$ meter equipped with a microelectrode (Hanna Instruments, Germany). The samples were left to equilibrate at room temperature for at least $24 \mathrm{~h}$ before measuring. Samples were stored for at most 1 week at a temperature of $4{ }^{\circ} \mathrm{C}$. For the spectroscopic experiments, solutions of high molecular weight hyaluronan in the putty state were prepared by 
dissolving $20 \mathrm{mg} / \mathrm{mL}$ hyaluronan sodium salt in heavy water $\left(\mathrm{D}_{2} \mathrm{O}\right)$ at a DCl concentration of $40 \mathrm{mM}$, which was previously determined to be the correct concentration to obtain the $\mathrm{pH}=2.5$ putty state conditions. ${ }^{39}$ We dissolved hyaluronan in $\mathrm{D}_{2} \mathrm{O}$ to facilitate the infrared observation of the amide and carbonyl stretch vibrations, which carry the structural information, at wavenumbers around 1630 and $1720 \mathrm{~cm}^{-1}$, respectively. Infrared measurements cannot be performed in $\mathrm{H}_{2} \mathrm{O}$ solutions, as the amide and carbonyl vibrations of hyaluronic acid absorb in the same infrared frequency region as the bending vibration of $\mathrm{H}_{2} \mathrm{O}$ (between 1600 and $1700 \mathrm{~cm}^{-1}$ ). The bending vibration of $\mathrm{D}_{2} \mathrm{O}$ is instead strongly red-shifted to 1200 $\mathrm{cm}^{-1}$. $^{43}$ Note that the $\mathrm{pD}$ value of $\mathrm{D}_{2} \mathrm{O}$ solution can be converted to the $\mathrm{pH}$ value of an $\mathrm{H}_{2} \mathrm{O}$ solution of similar acidity using $\mathrm{pH}=$ $\mathrm{pD}^{*} 0.929 .^{44}$

TTS Rheology Experiments. We measured the frequency spectra of the linear viscoelastic shear moduli as a function of temperature with a stress-controlled MCR 501 rheometer (Anton Paar, Austria) using a plate-plate measuring geometry with a $40 \mathrm{~mm}$ diameter and $100 \mu \mathrm{m}$ gap. The plate temperature was controlled by a Peltier plate connected to the lower plate and a hood controlling the temperature of the upper plate. Samples were loaded at room temperature $\left(22^{\circ} \mathrm{C}\right)$ and equilibrated for $10 \mathrm{~min}$ before starting the experiments. Small amplitude $(0.5 \%$ strain $)$ oscillatory shear tests were performed at 20 frequencies logarithmically spaced between 0.1 and $10 \mathrm{~Hz}$, at temperatures of $5,10,15,22,30$, and $40{ }^{\circ} \mathrm{C}$. We started at $22{ }^{\circ} \mathrm{C}$ and first decreased the temperature to 15,10 , and $5{ }^{\circ} \mathrm{C}$ and then increased the temperature to 30 and $40{ }^{\circ} \mathrm{C}$. The temperature was adjusted between each frequency sweep at the fastest rate allowed by the rheometer (around $2{ }^{\circ} \mathrm{C} / \mathrm{s}$ ), and after reaching the desired temperature, we waited for equilibration by waiting for the elastic and loss moduli to saturate, which always occurred within $2 \mathrm{~min}$. We verified that there was no hysteresis in the viscoelastic moduli upon cooling back to $22{ }^{\circ} \mathrm{C}$ until we increased the temperature to $60^{\circ} \mathrm{C}$ (Figure S1), consistent with prior work, ${ }^{45}$ that showed that hyaluronan is stable up to $50{ }^{\circ} \mathrm{C}$ and is hydrolyzed above $90{ }^{\circ} \mathrm{C}$ on a timescale of $\sim 1 \mathrm{~h}$. TTS analysis of the frequency $(\omega)$ spectra was performed by determining the shift factor $a_{T}(T)$ required to overlap both the elastic modulus $G^{\prime}(\omega)$ and the viscous shear modulus $G^{\prime \prime}(\omega)$ with their respective reference curves measured at the reference temperature $T_{0}=295 \mathrm{~K}\left(22^{\circ} \mathrm{C}\right)$. The temperature dependence of the shift factors $a_{\mathrm{T}}(T)$ was fitted to an Arrhenius equation to obtain the activation enthalpy $E_{\mathrm{a}}$ for stress relaxation

$$
\log \left(a_{\mathrm{T}}\right)=-\frac{E_{\mathrm{a}}}{2.303 R}\left(\frac{1}{T}-\frac{1}{T_{0}}\right)
$$

where $R$ is the universal gas constant. The activation energy in units of $k_{\mathrm{B}} T$ is obtained by dividing by Avogadro's number, where $k_{\mathrm{B}}$ is Boltzmann's constant and $T$ is the absolute temperature. At each temperature, we performed experiments on three independently prepared samples.

Linear Infrared Spectroscopy (FTIR). We measured Fourier Transform Infrared (FTIR) spectra of the samples with a Bruker Vertex 80v FTIR spectrometer equipped with a liquid-nitrogencooled mercury-cadmium-telluride (MCT) detector. The spectra were recorded under a nitrogen atmosphere at a wavenumber resolution of $3 \mathrm{~cm}^{-1}$. We averaged 100 scans for every spectrum. In all measurements, we used a standard sample cell with a path length of $100 \mu \mathrm{m}$. The temperature-dependent FTIR spectra were measured with the aid of a Peltier-cooled temperature cell (Mid-IR Falcon, Pike technologies). The temperature was ramped from 20 to $40{ }^{\circ} \mathrm{C}$ at a rate of $1{ }^{\circ} \mathrm{C} / \mathrm{min}$. The spectra were corrected for the absorption of the $\mathrm{D}_{2} \mathrm{O}$ solvent background at the same temperature. These background spectra were measured using the same ramping parameters. To determine if the ratio between the two protonation states of the carboxyl group depends on temperature, we fitted the linear infrared spectra measured at different temperatures using a global fitting procedure. This fit is based on the minimization of the square error

$$
\sum_{i, j}\left(S\left(\omega_{j}, T_{i}\right)-S^{\exp }\left(\omega_{j}, T_{i}\right)\right)^{2}
$$

where $S$ is the fitted spectrum, $S^{\exp }$ is the experimental spectrum, $\omega_{j}$ is the frequency, and $T_{i}$ is the temperature. Assuming that the vibrational bands are Gaussian shaped, we expect that

$$
\begin{aligned}
& \forall i, \text { for } \omega_{\{\min \}} \leq \omega_{j} \leq \omega_{\{\max \}} \\
& \boldsymbol{S}\left(\boldsymbol{\omega}_{j}, \quad \boldsymbol{T}_{i}\right)=\sum_{k=1,2,3} \boldsymbol{c}_{k}\left(\boldsymbol{T}_{i}\right) \boldsymbol{g}_{\boldsymbol{k}}\left(\boldsymbol{\omega}_{j}\right)+\boldsymbol{c}_{\boldsymbol{b k}}\left(\boldsymbol{T}_{\boldsymbol{i}}\right) \boldsymbol{S}_{\boldsymbol{b k}}\left(\boldsymbol{\omega}_{\mathbf{j}}\right)
\end{aligned}
$$

where $g_{k}$ represents the three Gaussians that describe the absorption bands of the antisymmetric stretching mode of the carboxylate anion $\left(\mathrm{COO}_{\mathrm{ant}}{ }^{-}\right)$, the amide I mode (AM.I), and the carbonyl stretching mode of the protonated carboxyl group $(\mathrm{C}=\mathrm{O})$, respectively, and $c_{k}$ represents the Gaussians amplitudes. $S_{b k}\left(\omega_{j}\right)$ represents the spectrum used for background correction. Based on previous work, ${ }^{39}$ the Gaussians were centered at 1609,1634 , and $1725 \mathrm{~cm}^{-1}$ for the absorption bands of the $\mathrm{COO}_{\text {ant }}{ }^{-}$, AM.I and $\mathrm{C}=\mathrm{O}$ vibrations, respectively. To account for the fact that increasing the temperature leads to a slight shift of the absorption bands, ${ }^{46}$ the center frequencies were allowed to shift by a maximum of $1 \mathrm{~cm}^{-1}$ when fitting the linear spectrum at $40{ }^{\circ} \mathrm{C}$. The widths of the three bands are global parameters in the fit, meaning that they are fixed at all studied temperatures and only the amplitudes of the three bands are allowed to be different at each temperature. We can then find the areas of the $\mathrm{COO}_{\text {ant }}{ }^{-}, \mathrm{AM} . \mathrm{I}$, and $\mathrm{C}=\mathrm{O}$ absorption bands at each temperature, and compare their ratio at different temperatures.

2DIR Spectroscopy. 2DIR measurements were performed using a home-built setup described elsewhere. ${ }^{47} \mathrm{We}$ excite the amide and carboxyl vibrations with a pair of strong femtosecond mid-infrared pulses centered at $1670 \mathrm{~cm}^{-1}$ with a bandwidth of $200 \mathrm{~cm}^{-1}(\sim 100 \mathrm{fs}$, $4 \mu \mathrm{J}$ per pulse). This excitation induces transient absorption changes that we probe with a weaker $(0.35 \mu \mathrm{J})$ single femtosecond probing pulse that is delayed by a waiting time $T_{\mathrm{w}}$. After propagation through the sample, the probe pulse was sent into an infrared spectrograph and detected with an infrared MCT detector array, thus yielding the transient absorption spectrum as a function of the probe frequency. The dependence of the transient absorption spectrum on the excitation frequency was determined by measuring transient spectra for many different delay times $\tau$ between the two excitation pulses. By Fourier transformation of these spectra, we obtain the dependence of the transient absorption spectrum on the excitation frequency. By plotting the transient absorption spectrum as a function of the excitation and the probing frequency, we obtain 2DIR transient absorption spectra for each delay time $T_{\mathrm{w}}$ between the excitation and probing pulses. The temperature of the sample was kept constant during the experiment with a Peltier element with an active feedback loop.

\section{RESULTS AND DISCUSSION}

pH Dependence of Hyaluronan Hydrogel Rheology. To study the impact of $\mathrm{pH}$-dependent cross-linking on hyaluronan hydrogel rheology, we compare solutions of hyaluronan polymers at $\mathrm{pH} 2.5$ and $\mathrm{pH} 7.0$ (see Figure 1a). These $\mathrm{pH}$ values were chosen because they represent two distinct regimes of hyaluronan chain interactions. At $\mathrm{pH}$ 7.0, previous rheology ${ }^{37}$ and self-diffusion ${ }^{26}$ measurements showed that hyaluronan chains interact by excluded volume interactions only, provided that the ionic strength of the solution is sufficiently high to screen the electrostatic repulsions of the chains. Nuclear magnetic resonance (NMR) and 2DIR ${ }^{33}$ measurements, ${ }^{48}$ compression measurements by atomic force microscopy (AFM) ${ }^{49}$ and molecular dynamic simulations of hyaluronan ${ }^{50}$ all showed that under neutral conditions, only intramolecular, no intermolecular, associative interactions are present. Specifically, NMR and 
(a)

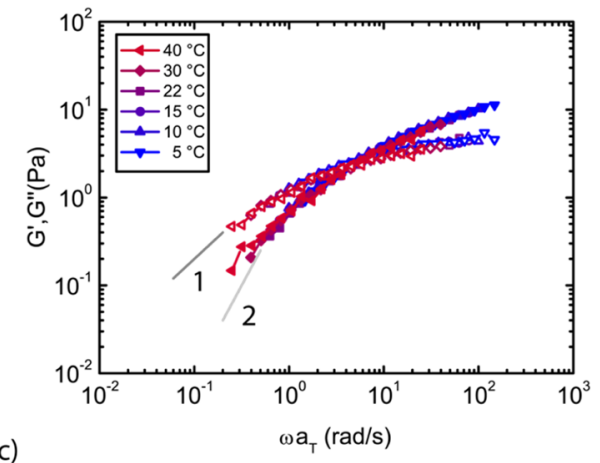

(c)

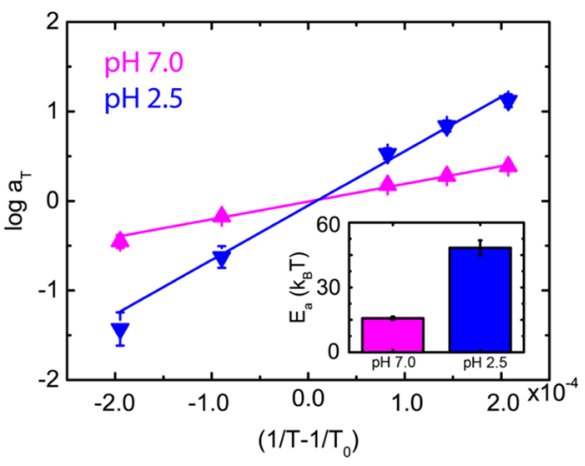

(b)
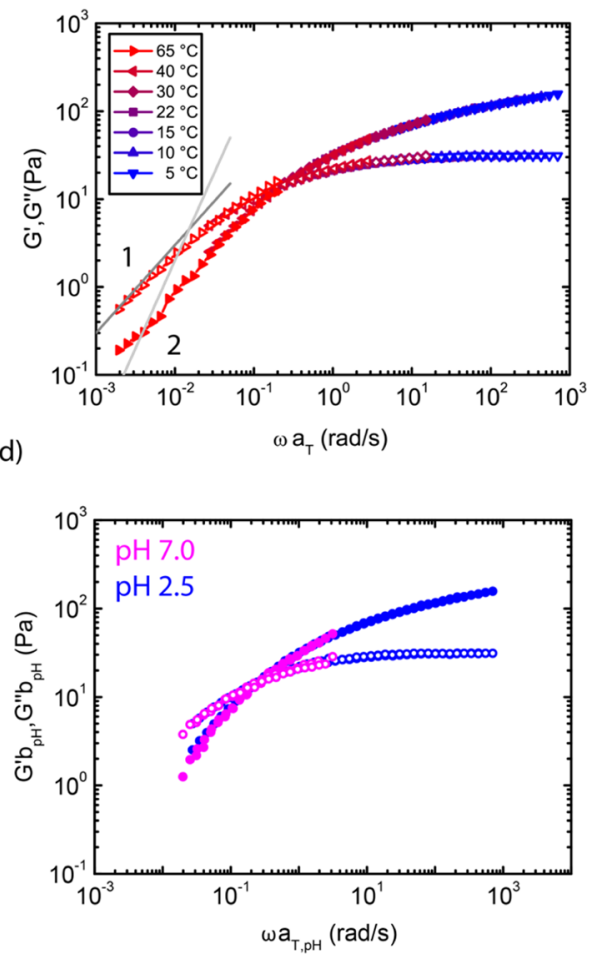

Figure 2. TTS rheology of hyaluronan solution $(10 \mathrm{mg} / \mathrm{mL})$. (a) Frequency sweeps at $\mathrm{pH} 7.0$ obtained at different temperatures are shifted along the frequency axis onto the measurement at the reference temperature of $22{ }^{\circ} \mathrm{C}$ by multiplication with a shift factor $a_{\mathrm{T}}$. (b) Corresponding rescaled frequency sweeps at $\mathrm{pH}$ 2.5. The gray lines in (a,b) indicate the expected terminal relaxation $G^{\prime \prime} \propto \omega^{1}$ and $G^{\prime} \propto \omega^{2}$ for a Maxwell fluid. (c) Arrhenius plot showing the shift factors used for constructing the TTS data as a function of the inverse of the temperature relative to the reference temperature. Lines show linear fits whose slopes represent the flow activation energy divided by $2.3 R$. Inset: Activation energies retrieved from the Arrhenius fits, expressed in units of thermal energy $k_{\mathrm{B}} T$. (d) Universal master curve for hyaluronan hydrogel rheology obtained by rescaling all frequency sweeps gathered at different temperatures $\left(5\right.$ and $40^{\circ} \mathrm{C}$, not color-coded) and $\mathrm{pH}$ values $(2.5$ and 7.0 , see legend) onto a reference set measured at $\mathrm{pH} 2.5$ and at $22{ }^{\circ} \mathrm{C}$ (blue triangles). We first shifted the horizontal axis and then the vertical axis to obtain overlap.

molecular dynamics simulations showed that a set of specific intramolecular hydrogen bonds, involving the hydroxyl groups of the sugar rings, is formed, increasing the chain stiffness of hyaluronan. ${ }^{48,50,51}$ Additionally, a small fraction $(\sim 10 \%)$ of carboxyl and amide groups of adjacent units form intramolecular hydrogen bonds. ${ }^{33}$ However, based on 2DIR experiments performed at different temperatures, we previously concluded that such intrachain interactions do not significantly contribute to the polymer rigidity. ${ }^{33}$ At $\mathrm{pH} \mathrm{7}$, there is no experimental evidence of the presence of interchain associative interactions. ${ }^{48}$ This molecular picture is also in line with several macroscopic studies, which showed that hyaluronan chain dynamics is dependent only on excluded volume interactions at $\mathrm{pH} 7 .^{52}$ At $\mathrm{pH} 2.5$, high molecular weight hyaluronan forms soft and stretchable gels that are referred to as putty. ${ }^{32,53}$ We recently showed with 2DIR spectroscopy measurements that this gel formation is driven by the $\mathrm{pH}$-dependent formation of hydrogen bonds involving carboxylic acid, the deprotonated carboxylate anion, and the amide groups of adjacent hyaluronan chains (Figure 1a). ${ }^{39} \mathrm{We}$ performed the rheology experiments on samples with a polymer concentration of $10 \mathrm{mg} / \mathrm{mL}$, in the semidilute entangled concentration regime. In this regime, the polymers experience transient topological constraints as a result of their high density. ${ }^{14}$ The entangled regime sets in at the entanglement concentration $c_{\mathrm{e}}$, which is generally a factor 25 higher than the overlap concentration $c^{*}$, where the polymer coils first touch. ${ }^{54}$ For hyaluronan with a molecular weight of
1.5-1.8 MDa, $c^{*} \approx 2 \mathrm{mg} / \mathrm{mL} .{ }^{55,56}$ We characterized the timedependent rheology of the samples by performing frequency sweeps using strain oscillations with a small (0.5\%) amplitude.

As shown in Figure $1 \mathrm{~b}$, the frequency spectra show a transition from a solid response (storage shear modulus $G^{\prime}$ larger than the loss modulus $\left.G^{\prime \prime}\right)$ at high frequencies to a fluid response $\left(G^{\prime}<G^{\prime \prime}\right)$ at low frequencies. This behavior is characteristic for entangled polymer solutions, where chains diffuse by reptation, that is, highly restricted diffusion because of entanglements with surrounding chains. ${ }^{54}$ Entangled polymer solutions generally display a Maxwell-type behavior with a rubber-like plateau that persists down to a frequency $\omega$ $\approx 1 / \tau$ and a terminal $(\omega<1 / \tau)$ regime, where the moduli tend toward $G^{\prime \prime} \propto \omega^{1}$ and $G^{\prime} \propto \omega^{2}$ scaling. ${ }^{54}$ The main effects of lowering the $\mathrm{pH}$ are first a downshift of the frequency, where $G^{\prime}$ and $G^{\prime \prime}$ crossover, which marks the transition from the rubber plateau to the terminal regime (from $5 \mathrm{rad} / \mathrm{s}$ at $\mathrm{pH} 7.0$ to $\sim 0.2 \mathrm{rad} / \mathrm{s}$ at $\mathrm{pH} 2.5$ ) and second an approximately 10 -fold increase of the elastic modulus. The rheology data do not reach high enough frequencies to access the plateau regime but indicate approximate values for the plateau modulus of $\sim 20 \mathrm{~Pa}$ at $\mathrm{pH} 7.0$ versus $\sim 200 \mathrm{~Pa}$ at $\mathrm{pH} 2.5$. These changes are as expected for entangled polymers that experience transient cross-linking interactions, as formulated by the sticky reptation model. ${ }^{34,35}$ This model predicts that sticky interactions slow down reptation by adding an additional friction on the chains that increases linearly with the (effective) bond lifetime and quadratically with the number of stickers per chain. The model 
further predicts that transient cross-links enhance the shear modulus $G_{0}$ in the rubbery plateau because $G_{0}$ is proportional to the number density of elastically active network strands $\nu_{\mathrm{x}}=$ $c N_{\mathrm{A}} / M$, according to $G_{0}=\nu_{\mathrm{x}} k_{\mathrm{B}} T$. Here, $N_{\mathrm{A}}$ is Avogadro's number and $M$ is the chain molecular weight. The magnitude of the elastic modulus is in line with what we expect at the hyaluronan concentration used here, which is just at the onset of entanglements $\left(c=5 c^{* 54}\right)$. The plateau modulus $G_{\mathrm{N}}$ of an entangled solution can be estimated according to $M_{\mathrm{e}}=c R T /$ $G_{\mathrm{N}}$, where $c$ is the polymer concentration and $M_{\mathrm{e}}$ is the entanglement or transient cross-linking molecular weight. We measure $G_{\mathrm{N}}$ values of around $20 \mathrm{~Pa}$ at $\mathrm{pH} 7.0$ and $200 \mathrm{~Pa}$ at $\mathrm{pH}$ 2.5. We caution that these values are approximations, as the rheology data do not access high enough frequencies such that the elastic modulus has truly reached its plateau value. By using $R=8.3[\mathrm{~Pa}]\left[\mathrm{m}^{3}\right]\left[\mathrm{K}^{-1}\right]\left[\mathrm{mol}^{-1}\right], c=10[\mathrm{mg} / \mathrm{mL}]=$ $104\left[\mathrm{~g} / \mathrm{m}^{3}\right], T=293[\mathrm{~K}]$, we therefore estimate $M_{\mathrm{e}}=1.2 \times 10^{6}$ and $1.2 \times 10^{5} \mathrm{~g} / \mathrm{mole}$ at $\mathrm{pH} 7.0$ and 2.5, respectively. At $\mathrm{pH} 7$, $M_{\mathrm{e}}$ is close to the average molecular weight $(1.5-1.8 \mathrm{MDa})$ of the polymers. At $\mathrm{pH}=2.5$, we compute a ratio $M / M_{\mathrm{e}} \approx 12-$ 15 , indicating around 11-14 effective cross-links per chain.

Temperature Dependence of Hyaluronan Hydrogel Rheology. Our findings suggest that the rheology of hyaluronan hydrogels is controlled by a single time scale, which is modulated by $\mathrm{pH}$-dependent chain interactions. To test this hypothesis, we performed measurements at different temperatures because temperature is known to change the time scale of hydrogen-bond breaking and reformation. ${ }^{57}$ As shown in Figure 1c,d, the shear moduli both at neutral ( $\mathrm{pH} 7.0)$ and at acidic ( $\mathrm{pH} \mathrm{2.5)} \mathrm{hyaluronan} \mathrm{solutions} \mathrm{strongly} \mathrm{depend} \mathrm{on}$ temperature. Increasing the temperature lowers the magnitude of the shear moduli and increases the characteristic frequency at which the rubber plateau crosses over to the terminal regime, consistent with faster stress relaxation. We note that the hyaluronan hydrogels were thermoreversible: there was no hysteresis in the rheology when the temperature was shifted back up or back down (Figure $S 1$ in the Supporting Information).

To test whether a single interaction mechanism can indeed account for the observed temperature dependence, we performed a TTS analysis using $22{ }^{\circ} \mathrm{C}$ as the reference temperature. The idea behind this analysis is that the stress relaxation time according to the sticky reptation model is proportional to the (effective) bond lifetime and should therefore be exponentially dependent on temperature. ${ }^{58}$ As shown in Figure 2, we can indeed construct master curves for both the $\mathrm{pH} 7.0$ data set (Figure 2a) and the $\mathrm{pH} 2.5$ data set (Figure $2 b$ ) by in each case shifting the data along the frequency axis by a temperature-dependent shift factor $a_{\mathrm{T}}$. The fact that the same horizontal shift factors apply for $G^{\prime}$ and $G^{\prime \prime}$ indicates that hyaluronan gels are thermorheologically simple materials $^{47}$ for which a single temperature-dependent interaction scale determines the rheology. The fact that we do not need any vertical (modulus) shift factor to superpose the frequency-dependent $G^{\prime} / G^{\prime \prime}$ curves suggests that the equilibrium density of cross-links does not change appreciably with temperature. The modulus depends on temperature through two effects, namely, through a direct linear proportionality with $k_{\mathrm{B}} T$ and through any temperature dependence of the cross-link density. Over the temperature range studied here $\left(5-40{ }^{\circ} \mathrm{C}\right)$, the $k_{\mathrm{B}} T$ term increases by only a factor of 1.1 . Thus, the observation that we do not need a vertical shift indicates that the change in number density of interchain hydrogens bonds with increasing temperature, if any, must be very small. The master curves clearly show Maxwell-type behavior with a rubbery plateau at the higher frequencies and a crossover to a terminal relaxation regime at lower frequencies. At $\mathrm{pH}$ 7.0, we do not reach sufficiently low frequencies to confirm whether the moduli at low frequencies tend toward the $G^{\prime \prime} \sim \omega^{1}$ and $G^{\prime}$ $\sim \omega^{2}$ dependencies expected for a Maxwell fluid with a single relaxation time. At $\mathrm{pH} 2.5$, we do reach lower normalized frequencies, but the low-frequency scaling of $G^{\prime}$ seems somewhat shallower than the $G^{\prime} \sim \omega^{2}$ scaling of the Maxwell model. A likely explanation is the size polydispersity of the hyaluronan, which is known to cause broadening of the frequency spectrum of entangled polymers, ${ }^{54}$ especially in the case of sticky polymers. ${ }^{41}$

To retrieve the $\mathrm{pH}$ dependence of the flow activation energy, we constructed Arrhenius plots of the shift factors $a_{\mathrm{T}}$ as a function of the inverse temperature relative to the reference temperature $T_{0}=22{ }^{\circ} \mathrm{C}$, both for $\mathrm{pH} 7.0$ and $\mathrm{pH} 2.5 .^{59}$ As shown in Figure $2 \mathrm{~b}$, we obtained straight lines at both $\mathrm{pH}$ values, indicating that the relaxation time is controlled by the thermally activated dissociation of bonds between chains. As shown in Figure 2c, the activation energy at $\mathrm{pH} 2.5$ (taken from the slope of the Arrhenius plots) is much higher than at $\mathrm{pH}$ 7.0, consistent with the expectation from the sticky reptation model that the formation of hydrogen bonds between the chains enhances the friction on the chains. In the entangled state at $\mathrm{pH} 7.0$, the activation energy is around $12 k_{\mathrm{B}} T$ (or $30 \mathrm{~kJ} / \mathrm{mol}$ ), consistent with prior findings. ${ }^{56} \mathrm{We}$ note that the earlier work was carried out at $50 \mathrm{mg} / \mathrm{mL}$, a much larger concentration than studied here $(10 \mathrm{mg} / \mathrm{mL})$, suggesting little effect of concentration on the activation energy. We also find little dependence of the activation energy on hyaluronan concentration at $\mathrm{pH} 7.0$ when we extend the concentration range down to $5 \mathrm{mg} / \mathrm{mL}$ (see Figure S2). For

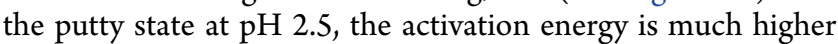
and amounts to $\sim 50 k_{\mathrm{B}} T$ (or $115 \mathrm{~kJ} / \mathrm{mol}$ ). We measured similar activation energies at other hyaluronan concentrations $(5-20 \mathrm{mg} / \mathrm{mL}$ ) (see Figure S2). The much higher flow activation energy at $\mathrm{pH} 2.5$ compared to $\mathrm{pH} 7.0$ is consistent with the additional presence of transient associations between the chains.

Until now, we rescaled the data sets for different $\mathrm{pH}$ values onto separate master curves. To test if a single constitutive law describes all data, independent of $\mathrm{pH}$ and temperature, we finally rescaled all curves onto a single master curve. This idea was inspired by recent studies showing that TTS can be extended to other environmental parameters that control the relaxation times of noncovalent gels such as ionic strength. ${ }^{60,61}$ We can indeed rescale the $G^{\prime}$ and $G^{\prime \prime}$ curves measured at $\mathrm{pH}$ 7.0 and $\mathrm{pH} 2.5$ onto a single universal set of master curves, using in addition to the horizontal shift factor $a_{\mathrm{T}}$ for the frequency axis also a vertical shift factor $b_{\mathrm{T}} \approx 1.5$. The collapse onto a single set of master curves strongly suggests that the physical mechanism that controls the rheology is the same, regardless of temperature and $\mathrm{pH}$.

Spectroscopic Measurement of the Density of Intermolecular Interactions. Prior 2DIR spectroscopy measurements from our laboratory ${ }^{39}$ showed that the crosslinks between hyaluronan chains are formed by hydrogen bonds between the carboxylic acid, deprotonated carboxylate anion, and amide groups of adjacent hyaluronan polymers (Figure 1a). The cross-link density thus likely depends on the degree of protonation of the carboxyl groups, which could in 
principle change with temperature. To test whether the protonation state of hyaluronan changes with temperature, we measured linear infrared absorption spectra of a solution of hyaluronan dissolved in $\mathrm{D}_{2} \mathrm{O}$ at 20 and $40{ }^{\circ} \mathrm{C}$ (Figure 3). The
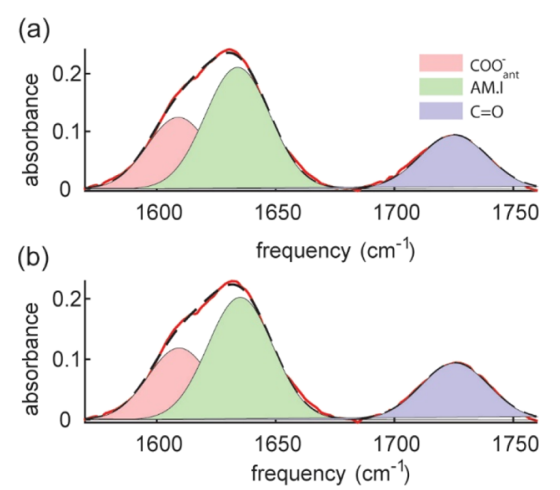

Figure 3. $(\mathrm{a}, \mathrm{b})$ Linear infrared spectra for a solution of hyaluronan of $20 \mathrm{mg} / \mathrm{mL}$ at $\mathrm{pH}=2.5$ at two temperatures of 20 and $40{ }^{\circ} \mathrm{C}$, respectively. Red solid lines represent the experimental data, while dashed black lines show the fits. By calculating the areas of the absorption bands, we find that the ratio between $-\mathrm{COO}^{-}$and $-\mathrm{COOH}$ is constant $(\sim 1.1)$.

solution was prepared at a concentration of $40 \mathrm{mM}$ of $\mathrm{DCl}$ in order to obtain a $\mathrm{pH}$ of 2.5 where hyaluronan is in the putty state, following the same protocol as previously described. ${ }^{39}$ We observe three absorption bands at 1633, 1725, and 1609 $\mathrm{cm}^{-1}$, which based on prior work ${ }^{62}$ we assign to, respectively, the amide I vibration (AM.I), the carbonyl stretching mode of the protonated carboxylic acid group $(\mathrm{C}=\mathrm{O})$, and the antisymmetric stretch vibration of the carboxylate group anion group $\left(\mathrm{COO}_{\mathrm{ant}}{ }^{-}\right)$. The relative intensities of the $\mathrm{C}=\mathrm{O}$ and $\mathrm{COO}_{\text {ant }}{ }^{-}$bands directly reflect the protonation state of hyaluronan. ${ }^{39}$ Using the procedure described in the Experimental Section, we determined the absorption band areas. We thus find that the fraction of deprotonated carboxyl groups $\left(-\mathrm{COO}^{-}\right)$is the same at 20 and at $40{ }^{\circ} \mathrm{C}$. From the acid-base equilibrium of hyaluronan, we calculate that $\sim 20 / 30 \%$ of the $-\mathrm{COO}^{-}$is deprotonated at $\mathrm{pH} 2.5$.

To measure the density of hydrogen bonds as a function of temperature, we performed 2DIR spectroscopy measurements on hyaluronan solutions in $\mathrm{D}_{2} \mathrm{O}$ at $\mathrm{pH}=2.5$ at several temperatures between 5 and $40{ }^{\circ} \mathrm{C}$ (Figure 4 ). We excited the amide and carboxyl vibrations with a strong $\sim 100$ femtosecond infrared pulse pair centered at $1670 \mathrm{~cm}^{-1}$. We probed the resulting transient absorption changes with a weaker $(0.35 \mu \mathrm{J})$ single femtosecond probing pulse that was delayed by a waiting time $T_{\mathrm{w}}$ (Figure $4 \mathrm{a}$ ). Figure $4 \mathrm{~b}, \mathrm{c}$ shows the isotropic 2DIR spectra at the two temperature extremes of 5 and $40{ }^{\circ} \mathrm{C}$. In both spectra, we observe on the diagonal four distinct spectral signatures. The blue-colored signatures represent negative absorption changes (which we refer to as bleach) that originate from a reduction of the absorption at the amide I and carboxyl carbonyl frequencies. This reduced absorption is due to the depletion of the fundamental transition $(v=0$ to $v=1)$ and stimulated emission $(v=1$ to $v=0)$ of the excited amide I and carbonyl vibrations. At somewhat lower probing frequencies, we observe red-colored spectral signatures, which represent positive absorption changes. These positive absorption changes can be assigned to excited-state absorption or esa and arise because of the vibrational transition from $v=1$ to $v=2 .^{63}$ The (a)

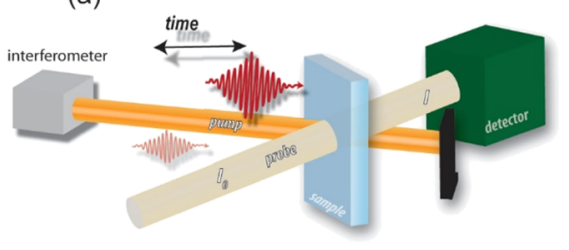

(b)
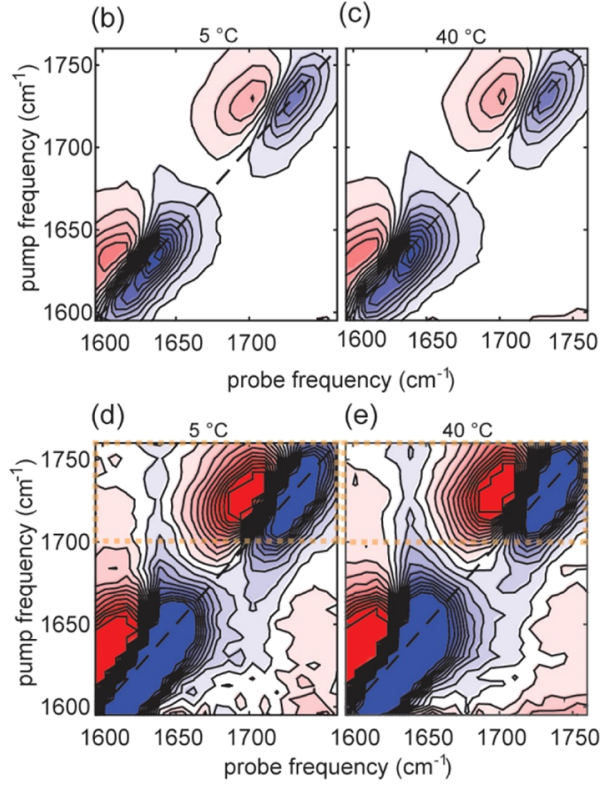

Figure 4. (a) Schematic of the 2DIR experiment (see main text for details). (b,c) 2DIR spectra of hyaluronan $(20 \mathrm{mg} / \mathrm{mL})$ at $\mathrm{pH}=2.5$ at a time delay $T_{\mathrm{w}}=0.3 \mathrm{ps}$ at two temperatures, namely, $5(\mathrm{~b})$ and $40{ }^{\circ} \mathrm{C}$ (c). Color code: from 1.5 a.u. (dark red) to -1.5 a.u. (dark blue) (b), from 0.75 a.u. (dark red) to -0.75 a.u. (dark blue). (d,e) Same spectra with color-bar fivefold magnified to show the presence of offdiagonal peaks in the frequency regions indicated by the orange dashed rectangles.

two pairs of diagonal peaks are obtained by exciting at 1633 and $1725 \mathrm{~cm}^{-1}$, corresponding to the vibrational frequencies of the amide $\mathrm{I}$ and $\mathrm{C}=\mathrm{O}$ absorption bands, respectively. The lower-frequency diagonal peak shows a shoulder at $1610 \mathrm{~cm}^{-1}$, which we assign to the antisymmetric stretch vibration of the carboxylate anion.

When we multiply the 2DIR spectra by a factor of 5 , we can also observe off-diagonal spectral signatures in the 2DIR spectrum at both temperatures (Figure $4 \mathrm{~d}, \mathrm{e}$ ). The off-diagonal peaks are 10 -fold weaker than the diagonal signals. In the topleft off-diagonal region (exciting around $1725 \mathrm{~cm}^{-1}$ and probing around $1630 \mathrm{~cm}^{-1}$ ), which is indicated by the orangecolored rectangle, we observe a 2DIR absorption signal associated with the excitation of the carbonyl vibration and probing of the amide I absorption. This off-diagonal feature is commonly referred to as a downhill cross-peak. Figure 5a reports the 2DIR signals obtained by averaging over the excitation frequencies between 1700 and $1760 \mathrm{~cm}^{-1}$ (orange rectangle in Figure $4 \mathrm{~d}, \mathrm{e})$. At each temperature, the 2DIR signal is normalized to the maximum intensity of the carbonyl bleach. In Figure 5a, we observe again the bleach and the esa of the carbonyl around $1700 \mathrm{~cm}^{-1}$ and a negative 2DIR signal near $1635 \mathrm{~cm}^{-1}$, which is the absorption frequency of the amide I vibration. The cross-peak signal between the carbonyl stretching and the amide I vibrations reveals the existence of molecular coupling between these groups at $\mathrm{pH}=2.5$ and 
(a)

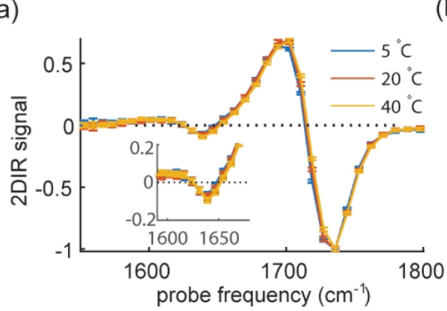

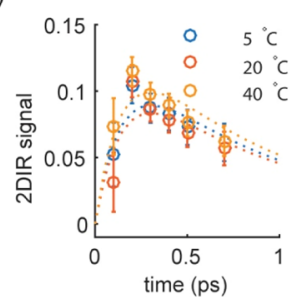

(c)

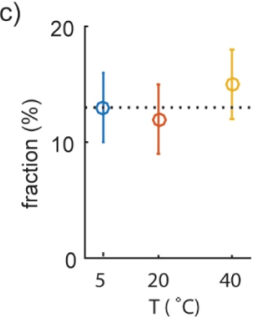

Figure 5. Temperature dependence of 2DIR spectral features for hyaluronan hydrogels at $\mathrm{pH}=2.5$. (a) $2 \mathrm{DIR}$ signals at three different temperatures obtained by averaging over the pump region between 1700 and $1760 \mathrm{~cm}^{-1}$. Inset: details of the $2 \mathrm{DIR}$ signal in the absorption frequency of the amide I vibration showing the cross-peak signal is independent of temperature. (b) Cross-peak decay traces at 5 , 20 , and $40{ }^{\circ} \mathrm{C}$. Dashed lines are fits to the relaxation model described in the main text. (c) Fraction of hydrogen-bonded carboxylic acid groups extracted from the 2DIR data is independent of temperature.

shows that a fraction of the carboxyl and the amide I groups are connected by a hydrogen bond.

To extract the fraction of hydrogen-bonded carboxyl and amide I groups, we consider the delay-time traces of the crosspeak signals shown in Figure 5b, obtained by fitting the 2DIR signals reported in Figure 5a (see the Methods section in the Supporting Information for details). The three delay-time traces are very similar, not showing a significant change upon changing the temperature. In order to extract the fraction of the hydrogen-bonded carboxyl and amide I groups at different temperatures, we analyzed the delay-time traces. In an earlier 2DIR study of hyaluronan, ${ }^{39}$ we showed that upon excitation, the carbonyl stretching mode can relax following two different relaxation paths.

A fraction of the excited $\mathrm{C}=\mathrm{O}$ vibrations relaxes by fast energy transfer to the amide I vibration with a time constant $T_{\text {ent }}(\sim 0.150 \mathrm{ps})$. This fast relaxation is enabled by the formation of the intermolecular hydrogen bonds. This energytransfer process leads to excitation of the amide I vibration and thus to a fast rise of the cross-peak signal. Subsequently, the amide I mode relaxes back to the ground state with a time constant $T_{1 \text { AM.I }}(\sim 0.65 \mathrm{ps})$. Another fraction of the excited $\mathrm{C}=\mathrm{O}$ groups is not hydrogen-bonded to an amide group and relaxes to low-frequency modes of the solvent or the hyaluronan polymer chain. This process is much slower and occurs with a time constant $T_{1 \mathrm{COOD}}(\sim 0.7 \mathrm{ps})$. Once populated, the low-frequency modes affect the absorption spectrum of the amide I vibration and also generate a 2DIR cross-peak signal. This latter contribution to the signal decays with a time constant $T_{1 \mathrm{LFM}}$, which is the relaxation time of the low-frequency modes. In our previous work, ${ }^{39}$ we found that $T_{1 \text { LFM }}$ is $\sim 3 \mathrm{ps}$, which is much slower than the decay of the amide $\mathrm{I}$ and $\mathrm{C}=\mathrm{O}$ vibrations. Because the two relaxation mechanisms that determine the cross-peak dynamics occur on quite different time scales, they can be distinguished in a fit of the cross-peak delay-time traces with the following expression:

$$
\begin{aligned}
\frac{\Delta \alpha_{\mathrm{CP}}\left(T_{\mathrm{w}}\right)}{\Delta \alpha_{\mathrm{COOD}}\left(T_{\mathrm{w}}\right)}= & c_{\text {anh }}\left(\frac{T_{1 \mathrm{LFM}}}{T_{1 \text { COOD }}-T_{1 \mathrm{LFM}}}\right)\left(\mathrm{e}^{-T_{\mathrm{w}} / T_{1 \text { COOD }}}\right. \\
& \left.-\mathrm{e}^{-T_{\mathrm{w}} / T_{\text {LIFM }}}\right)+c_{\text {ent }}\left(\frac{T_{1 \text { AM.I }}}{T_{\text {ent }}-T_{1 \text { AM.I }}}\right) \\
& \left(\mathrm{e}^{-T_{\mathrm{w}} / T_{\text {ent }}}-\mathrm{e}^{-T_{\mathrm{w}} / T_{\text {IAM.I }}}\right)
\end{aligned}
$$

where $\Delta \alpha_{\mathrm{CP}}\left(T_{\mathrm{w}}\right) / \Delta \alpha_{\mathrm{COOD}}\left(\overline{T_{\mathrm{w}}}\right)$ is the intensity of the crosspeak normalized to the intensity of the $\mathrm{C}=\mathrm{O}$ vibration at early time delay and $\overline{T_{\mathrm{w}}}=200 \mathrm{fs}$. The parameters $c_{\mathrm{anh}}$ and $c_{\mathrm{ent}}$ represent the cross-peak amplitudes associated with the anharmonic coupling and direct energy-transfer mechanisms, respectively, and are the only free parameters. Because of the limited amount of data points at long time delay, the value of $c_{\text {anh }}$ is constrained to be at least 0.02 , which is the minimum value expected for this parameter based on our previous work $\left(c_{\mathrm{anh}}=0.025 \pm 0.005\right)$. Hence, we extract the amplitude of the fast relaxation component because of energy transfer, which can be directly linked to the fraction of protonated carboxylic acid bonded to the amide groups. We find that around 15\% of the carbonyl groups are hydrogen-bonded to the amide groups at $\mathrm{pH}=2.5$ (Figure $5 \mathrm{C}$ ), which closely agrees with our previous findings from 2DIR spectroscopy. ${ }^{39}$ The requirement to alternate a double bond between a $-\mathrm{COOH}$ and amide with a single bond between $-\mathrm{COO}^{-}$and amide (as observed in Figure 1a) strongly limits the fraction of carboxylic acid groups that sterically can form a double hydrogen bond with an amide group. As shown in Figure 5c, we also observed that this fraction does not change in the temperature interval between 5 and $40{ }^{\circ} \mathrm{C}$, which indicates that for these carboxylic acid groups the free energy of a configuration with a double hydrogen bond to an amide group is similar to the free energy of a configuration in which the carboxylic acid group is hydrogen-bonded to water molecules. This would mean that the total fraction of carboxylic acid groups that sterically can engage in a double hydrogen bond with an amide group is $\sim 30 \%$.

Connection between Rheology and 2DIR. The 2DIR measurements show that the fraction of carboxyl groups that are engaged in a direct hydrogen bond with an amide group does not change with temperature between 5 and $40{ }^{\circ} \mathrm{C}$. This finding is consistent with the TTS analysis of the rheology data, which showed that the density of stickers that impede stress relaxation also does not change with temperature. These findings indicate that the breaking of an interchain hydrogen bond between a carboxyl group and an amide group results in a state that has about the same overall free energy as the intact hydrogen bond (see schematic in Figure 6). This other state is probably formed by hydrogen bonds between the carboxyl and the amide groups and solvent molecules. As a result, a change in temperature does not affect the number of hydrogen bonds that are formed between the polymer chains. However, the kinetics do change with temperature, as the breaking and reformation involves an energy barrier $E_{\mathrm{A}, \mathrm{hb}}$ that will be of the order of the binding energy of a single hydrogen bond, which is typically $\sim 400-1400 \mathrm{~cm}^{-1}$ or $2-7 k_{\mathrm{B}} T(\mathrm{~T}=298 \mathrm{~K}){ }^{64,65}$

To understand the acceleration of the network dynamics with increasing temperature, we need to find the relation between the characteristic lifetime of a single hydrogen bond $\tau$ 
(a)

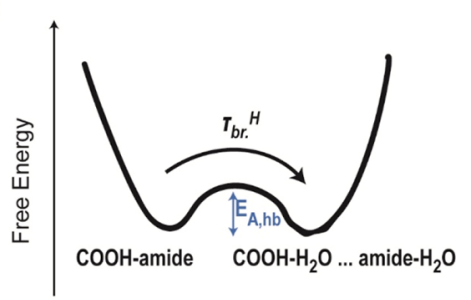

(b)

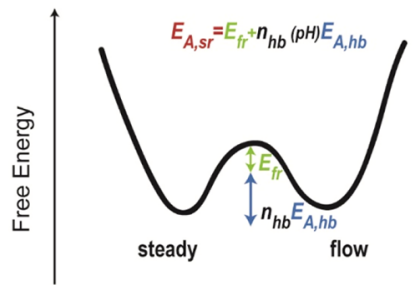

Figure 6. (a) Schematic representation of the breaking of a hydrogenbonded complex. We refer to the breakage time of the hydrogen bonds as $\tau_{\mathrm{br}}^{\mathrm{H}}$. The activation energy necessary to break the hydrogenbonded complex is defined as $E_{\mathrm{A}, \mathrm{hb}}$. (b) Schematic representation of the network dynamics. The flow activation energy $E_{\mathrm{A}, \mathrm{sr}}$ corresponds to the breaking of $n_{\mathrm{hb}}$ hydrogen bonds (stickers).

and the characteristic time scale of the stress relaxation of the hyaluronan solution, as expressed in the frequency dependence of the shear moduli. The typical lifetime $\tau=\tau_{\mathrm{br}}^{\mathrm{H}}$ of a single hydrogen bond is on the order of a few to tens of picoseconds. ${ }^{36}$ This large disparity in time scales between single hydrogen bond dynamics and sticker dynamics can be understood from the fact that multiple hydrogen bonds $n_{\mathrm{hb}}$ need to be broken at the same time to allow for chain displacement and thus flow of the network. In addition, such a mesoscopic rearrangement involves a frictional barrier $E_{\mathrm{fr}}$ because of chain entanglements and viscous drag with the solvent. Hence, the activation energy necessary for the network to flow will be larger than the activation energy of breaking a single hydrogen bond. Assuming a similar pre-exponential (Arrhenius) prefactor for the breaking of hydrogen bonds and the friction, the total activation energy for stress relaxation can be written as

$$
E_{\mathrm{A}, \mathrm{sr}}(\mathrm{pH})=n_{\mathrm{hb}}(\mathrm{pH}) E_{\mathrm{A}, \mathrm{hb}}+E_{\mathrm{fr}}
$$

At $\mathrm{pH}=7$, we can assume that there are no hydrogen bonds $\left(n_{\mathrm{hb}}=0\right)$ formed between the hyaluronan polymer chains based on earlier work showing that excluded volume interactions alone are enough to explain the concentrationdependent rheology. ${ }^{37}$ Thus, the activation energy at $\mathrm{pH} 7$ will be equal to the frictional barrier energy $E_{\mathrm{fr}}$. At $\mathrm{pH}=2.5$, hydrogen bonds are formed between the hyaluronan polymer chains and their density is large enough to cause the formation of an elastic hydrogel. By subtracting the frictional energy $E_{\mathrm{fr}}$ from the activation energy measured at $\mathrm{pH}=2.5, E_{\mathrm{A}, \mathrm{sr}}$, we find that the energy barrier due to hydrogen bonds at $\mathrm{pH} 2.5$ is around $85 \mathrm{~kJ} / \mathrm{mol}\left(\sim 38 k_{\mathrm{B}} T\right)$. This residual activation energy corresponds to $n_{\mathrm{hb}}=5-15$ hydrogen bonds, which thus need to be broken in order for the hyaluronan chain to diffuse at $\mathrm{pH}$ $=2.5$.

An alternative way to estimate the number of hydrogen bonds that need to be simultaneously dissociated to enable stress relaxation is to consider the number of hydrogen bonds belonging to the same Kuhn segment. ${ }^{66}$ Based on previous literature, ${ }^{67}$ the Kuhn length $\left(L_{\mathrm{k}}\right)$ of hyaluronan is $\sim 18 \mathrm{~nm}$ at
$200 \mathrm{mM} \mathrm{NaCl}$ at neutral $\mathrm{pH}$, which is consistent with the highest estimation reported in the literature for the persistence length $\left(L_{\mathrm{p}}=7-10 \mathrm{~nm}\right)^{33}$ assuming a worm-like chain model $\left(L_{\mathrm{k}}=2 L_{\mathrm{p}}\right)$. In AFM measurements, ${ }^{50}$ we recently measured a persistence length of $\sim 4 \mathrm{~nm}$ (and thus $L_{\mathrm{k}}=8 \mathrm{~nm}$ ) under similar conditions. Persistence length measurements at $\mathrm{pH}=$ 2.5 are unfortunately not available, but we expect a similar value because there is still a significant presence of negative charges at this $\mathrm{pH}$. Assuming a Kuhn length of $8 \mathrm{~nm}$, we estimate that $\sim 8$ disaccharide units belong to the same $L_{\mathrm{k}}$ segment. Further, we previously demonstrated ${ }^{39}$ that an alternation of $\mathrm{COOH}$ and $\mathrm{COO}^{-}$groups is necessary for the formation of a strong hydrogen-bond network (see Figure 1a of the article). Considering that from steric considerations, $\sim 30 \%$ of the carboxylic acid groups can form a hydrogen bond complex with an amide group on an adjacent chain and that half of these groups, that is, $\sim 15 \%$ of the total number of carboxylic acid groups, is actually involved in the hydrogenbond formation, we find that per Kuhn's segment around one $-\mathrm{COOH}$ forms a double hydrogen bond with the amide group of a second chain, and similarly per Kuhn's segment around one amide group forms a double bond with the $-\mathrm{COOH}$ group of a second chain, leading to four hydrogen bonds per chain. Furthermore, per Kuhn's segment around two $-\mathrm{COO}^{-}$ groups can form hydrogen bonds with the amide groups of a second chain, and similarly per Kuhn's segment around two amide groups can form hydrogen bonds with the $-\mathrm{COO}^{-}$ groups of a second chain, leading to an additional four hydrogen bonds. We thus estimate that $\sim 8$ hydrogen bonds can be formed per Kuhn's segment. This number agrees well with the estimated number of hydrogen bonds $n_{\mathrm{hb}} \approx 5-15$ per transient cross-link, which we estimated from the flow activation energy.

Furthermore, from the plateau modulus and the polymer concentration, we estimated earlier that the number of active transient cross-links is $11-14$. By assuming that every crosslink point is composed of 5-15 hydrogen bonds, as we infer from the activation enthalpy, we find that the total number of hydrogen bonds per chain $n_{\mathrm{hb}}$ is around 55-210. This estimate is consistent with the finding from the 2DIR measurements that the number of hydrogen bonds per chain is roughly 375550 , assuming that one single chain is composed by roughly 3750 units and that just $10-15 \%$ of these participate in hydrogen-bond formation. We conclude that the number of hydrogen bonds that we estimated from the 2DIR data is consistent with the corresponding number extracted from the rheology data.

Finally, it should be noted that we have not considered the possible effect of temperature on the frictional energy barrier $E_{\text {fr. }}$. This energy barrier will depend on several parameters, such as the intramolecular hydrogen bonds that stiffen hyaluronan and the mobility of the solvent molecules. Increased chain flexibility and water mobility could potentially lower $E_{\mathrm{fr}}$ as the temperature increases. ${ }^{68,69}$ However, the fact that the temperature dependence of the shift factor $a_{\mathrm{T}}$ can be well described with an Arrhenius expression suggests that the value of $E_{\mathrm{fr}}$ is quite insensitive to temperature changes within the range (5$40{ }^{\circ} \mathrm{C}$ ) we studied.

\section{CONCLUSIONS}

We used rheological and 2DIR spectroscopy measurements to measure the effect of temperature and $\mathrm{pH}$ on the mechanical properties and underlying molecular interactions of entangled 
hyaluronan polymers. The rheology results showed that changing the $\mathrm{pH}$ from 7.0 to 2.5 introduces associative interactions that strongly slow down stress relaxation. The 2DIR results showed that these associative interactions are mediated by hydrogen bonds whose equilibrium density does not depend on the temperature (from 5 to $40^{\circ} \mathrm{C}$ ) but only on $\mathrm{pH}$. Hence, the equilibrium structure of the hyaluronan polymer network does not change with temperature. The kinetics of the polymer network does show a strong dependence on temperature. By performing measurements over an extended temperature range $\left(5-40{ }^{\circ} \mathrm{C}\right)$, we could show that the stress relaxation time displays an Arrhenius-type dependence on temperature with a flow activation energy of 12 $k_{\mathrm{B}} T(\sim 30 \mathrm{~kJ} / \mathrm{mol})$ at $\mathrm{pH} 7.0$ and $50 k_{\mathrm{B}} T(\sim 115 \mathrm{~kJ} / \mathrm{mol})$ at $\mathrm{pH}$ 2.5. The activation energy at $\mathrm{pH} 7.0$ can be assigned to a frictional energy barrier associated with the motion of the polymer chains along each other and the displacement of water molecules. The much higher activation energy at $\mathrm{pH}=2.5$ indicates that under these conditions, the simultaneous breaking of 5-15 hydrogen bonds is required for chains to move and, thus, for the network to flow. Our findings show that hyaluronan forms thermoreversible gels whose macroscopic properties can be tuned by $\mathrm{pH}$ and temperature.

In the context of human physiology, understanding how the mechanical properties of extracellular glycosaminoglycans such as hyaluronan are affected by $\mathrm{pH}$ is relevant because many diseases, such as cancer and inflammation, are associated with acidosis, a decrease of the extracellular $\mathrm{pH} .{ }^{70-72}$ Even though the average $\mathrm{pH}$ values in this situation are reported to be of order 5.8 [ref 73], potentially lower $\mathrm{pH}$ values might be reached locally and transiently, which may strongly affect the dynamics of the hyaluronan polymer network. Moreover, $\mathrm{pH}$ responsive hydrogels may potentially serve for targeted release of anticancer drugs. ${ }^{74}$ In the context of materials science, it has become clear in recent years that noncovalently bonded hydrogels have many desirable properties such as stimulusresponsiveness, toughness, and an inherent capacity for selfhealing and recovery after mechanical damage. Such materials have possible applications for shape memory, self-healing, and adhesive materials. ${ }^{1}$ Hyaluronan is biocompatible and already used for a broad range of biomedical applications, from drug delivery to tissue regeneration. ${ }^{7,8,75}$ It would therefore be interesting to extend our study of the linear rheological properties of hyaluronan hydrogels in the future to the nonlinear regime of large deformations and to complex timedependent effects such as plasticity and self-healing. ${ }^{76}$

\section{ASSOCIATED CONTENT}

\section{sI Supporting Information}

The Supporting Information is available free of charge at https://pubs.acs.org/doi/10.1021/acs.macromol.0c01742.

Fitting procedure of the transient absorption spectra and the relaxation model and thermoreversibility of the rheology of hyaluronan and the concentration dependence of the flow activation energies (PDF)

\section{AUTHOR INFORMATION}

\section{Corresponding Authors}

Gijsje H. Koenderink - AMOLF, Amsterdam 1098 XG, The Netherlands; Department of Bionanoscience, Kavli Institute of Nanoscience Delft, Delft University of Technology, Delft 2629
HZ, The Netherlands; 이이이.org/0000-0002-7823-8807; Email: g.h.koenderink@tudelft.nl

Huib J. Bakker - AMOLF, Amsterdam 1098 XG, The Netherlands; $\odot$ orcid.org/0000-0003-1564-5314; Email: h.bakker@amolf.nl

\section{Authors}

Giulia Giubertoni - AMOLF, Amsterdam 1098 XG, The Netherlands; $\odot$ orcid.org/0000-0002-3417-4987

Federica Burla - AMOLF, Amsterdam 1098 XG, The Netherlands

Complete contact information is available at:

https://pubs.acs.org/10.1021/acs.macromol.0c01742

\section{Notes}

The authors declare no competing financial interest.

\section{ACKNOWLEDGMENTS}

We thank Fred MacKintosh (Rice University, Texas) for useful discussions on polymer rheology and Oleksandr O. Sofronov (AMOLF, The Netherlands) for useful experimental suggestions. This work was funded by the Netherlands Organisation for Scientific Research (NWO, grant number FOM-i41) and was part of the Industrial Partnership Programme Hybrid Soft Materials that is carried out under an agreement between Unilever Research and Development B.V. and NWO.

\section{REFERENCES}

(1) Cohen Stuart, M. A.; Huck, W. T. S.; Genzer, J.; Müller, M.; Ober, C.; Stamm, M.; Sukhorukov, G. B.; Szleifer, I.; Tsukruk, V. V.; Urban, M.; Winnik, F.; Zauscher, S.; Luzinov, I.; Minko, S. Emerging applications of stimuli-responsive polymer materials. Nat. Mater. 2010, 9, 101-113.

(2) Zhang, Z.; Chen, Q.; Colby, R. H. Dynamics of associative polymers. Soft Matter 2018, 14, 2961-2977.

(3) Hofman, A. H.; van Hees, I. A.; Yang, J.; Kamperman, M. Bioinspired Underwater Adhesives by Using the Supramolecular Toolbox. Adv. Mater. 2018, 30, 1704640.

(4) Li, J.; Mooney, D. J. Designing hydrogels for controlled drug delivery. Nat. Rev. Mater. 2016, 1, 16071.

(5) Rosales, A. M.; Anseth, K. S. The design of reversible hydrogels to capture extracellular matrix dynamics. Nat. Rev. Mater. 2016, 1, 15012.

(6) Stanton, M. M.; Samitier, J.; Sánchez, S. Bioprinting of 3D hydrogels. Lab-on-a-Chip 2015, 15, 3111-3115.

(7) Highley, C. B.; Prestwich, G. D.; Burdick, J. A. Recent advances in hyaluronic acid hydrogels for biomedical applications. Curr. Opin. Biotechnol. 2016, 40, 35-40.

(8) Wolf, K. J.; Kumar, S. Hyaluronic Acid: Incorporating the Bio into the Material. ACS Biomater. Sci. Eng. 2019, 5, 3753-3765.

(9) Geissler, E.; Hecht, A. M.; Horkay, F. Scaling equations for a biopolymer in salt solution. Phys. Rev. Lett. 2007, 99, 267801.

(10) Berezney, J. P.; Saleh, O. A. Electrostatic effects on the conformation and elasticity of hyaluronic acid, a moderately flexible polyelectrolyte. Macromolecules 2017, 50, 1085-1089.

(11) Lee, J. Y.; Spicer, A. P. Hyaluronan: a multifunctional, megaDalton, stealth molecule. Curr. Opin. Cell Biol. 2000, 12, 581586.

(12) Horkay, F.; Basser, P. J.; Londono, D. J.; Hecht, A.-M.; Geissler, E. Ions in hyaluronic acid solutions. J. Chem. Phys. 2009, 131, 184902.

(13) Horkay, F.; Magda, J.; Alcoutlabi, M.; Atzet, S.; Zarembinski, T. Structural, mechanical and osmotic properties of injectable hyaluronan-based composite hydrogels. Polymer 2010, 51, 4424-4430.

(14) Cowman, M. K.; Schmidt, T. A.; Raghavan, P.; Stecco, A. Viscoelastic properties of hyaluronan in physiological conditions. F1000Research 2015, 4, 622. 
(15) Zhu, Y.; Granick, S. Biolubrication: Hyaluronic Acid and the Influence on Its Interfacial Viscosity of an Antiinflammatory Drug. Macromolecules 2003, 36, 973-976.

(16) Shenoy, V.; Rosenblatt, J. Diffusion of macromolecules in collagen and hyaluronic acid, rigid-rod-flexible polymer, composite matrixes. Macromolecules 1995, 28, 8751-8758.

(17) Richter, R. P.; Baranova, N. S.; Day, A. J.; Kwok, J. C. Glycosaminoglycans in extracellular matrix organisation: are concepts from soft matter physics key to understanding the formation of perineuronal nets? Curr. Opin. Struct. Biol. 2018, 50, 65-74.

(18) Kuo, J. C.-H.; Gandhi, J. G.; Zia, R. N.; Paszek, M. J. Physical biology of the cancer cell glycocalyx. Nat. Phys. 2018, 14, 658-669.

(19) Neuman, M. G.; Nanau, R. M.; Oruña-Sanchez, L.; Coto, G. Hyaluronic acid and wound healing. J. Pharm. Pharm. Sci. 2015, 18, $53-60$.

(20) Rooney, P.; Kumar, S. Inverse relationship between hyaluronan and collagens in development and angiogenesis. Differentiation 1993, 54, 1-9.

(21) Petrey, A. C.; de la Motte, C. A. Hyaluronan, a crucial regulator of inflammation. Front. Immunol. 2014, 5, 101.

(22) Back, S. A.; Tuohy, T. M. F.; Chen, H.; Wallingford, N.; Craig, A.; Struve, J.; Luo, N. L.; Banine, F.; Liu, Y.; Chang, A.; Trapp, B. D.; Bebo, B. F., Jr.; Rao, M. S.; Sherman, L. S. Hyaluronan accumulates in demyelinated lesions and inhibits oligodendrocyte progenitor maturation. Nat. Med. 2005, 11, 966-972.

(23) Liu, M.; Tolg, C.; Turley, E. Dissecting the Dual Nature of Hyaluronan in the Tumor Microenvironment. Front. Immunol. 2019, 10, 947.

(24) Rankin, K. S.; Frankel, D. Hyaluronan in cancer - from the naked mole rat to nanoparticle therapy. Soft Matter 2016, 12, 38413848.

(25) Temple-Wong, M. M.; Ren, S.; Quach, P.; Hansen, B. C.; Chen, A. C.; Hasegawa, A.; D’Lima, D. D.; Koziol, J.; Masuda, K.; Lotz, M. K.; Sah, R. L. Hyaluronan concentration and size distribution in human knee synovial fluid: variations with age and cartilage degeneration. Arthritis Res. Ther. 2016, 18, 18.

(26) Gribbon, P.; Heng, B. C.; Hardingham, T. E. The molecular basis of the solution properties of hyaluronan investigated by confocal fluorescence recovery after photobleaching. Biophys. J. 1999, 77, $2210-2216$.

(27) Innes-Gold, S. N.; Pincus, P. A.; Stevens, M. J.; Saleh, O. A. Polyelectrolyte Conformation Controlled by a Trivalent-Rich Ion Jacket. Phys. Rev. Lett. 2019, 123, 187801.

(28) Gibbs, D. A.; Merrill, E. W.; Smith, K. A.; Balazs, E. A. Rheology of hyaluronic acid. Biopolymers 1968, 6, 777-791.

(29) Gatej, I.; Popa, M.; Rinaudo, M. Role of the $\mathrm{pH}$ on hyaluronan behavior in aqueous solution. Biomacromolecules 2005, 6, 61-67.

(30) Wu, S.; Ai, L.; Chen, J.; Kang, J.; Cui, S. W. Study of the mechanism of formation of hyaluronan putty at $\mathrm{pH}$ 2.5: part I. Experimental measurements. Carbohydr. Polym. 2013, 98, 16771682.

(31) Wu, S.; Ai, L.; Chen, J.; Kang, J.; Cui, S. W. Study of the mechanism of formation of hyaluronan putty at $\mathrm{pH}$ 2.5: Part IITheoretical analysis. Carbohydr. Polym. 2013, 98, 1683-1688.

(32) Balazs, E. A.; Cui, J. The story of "Hyaluronan Putty". Bioact. Carbohydr. Diet. Fibre 2013, 2, 143-151.

(33) Giubertoni, G.; Koenderink, G. H.; Bakker, H. J. Direct Observation of Intrachain Hydrogen Bonds in Aqueous Hyaluronan. J. Phys. Chem. A 2019, 123, 8220-8225.

(34) Leibler, L.; Rubinstein, M.; Colby, R. H. Dynamics of reversible networks. Macromolecules 1991, 24, 4701-4707.

(35) Rubinstein, M.; Semenov, A. N. Dynamics of entangled solutions of associating polymers. Macromolecules 2001, 34, 10581068.

(36) Woutersen, S.; Mu, Y.; Stock, G.; Hamm, P. Hydrogen-bond lifetime measured by time-resolved 2D-IR spectroscopy: N-methylacetamide in methanol. Chem. Phys. 2001, 266, 137-147.
(37) Krause, W. E.; Bellomo, E. G.; Colby, R. H. Rheology of sodium hyaluronate under physiological conditions. Biomacromolecules 2001, 2, 65-69.

(38) Charlot, A.; Auzély-Velty, R. Novel hyaluronic acid based supramolecular assemblies stabilized by multivalent specific interactions: rheological behavior in aqueous solution. Macromolecules 2007, 40, 9555-9563.

(39) Giubertoni, G.; Burla, F.; Martinez-Torres, C.; Dutta, B.; Pletikapic, G.; Pelan, E.; Rezus, Y. L. A.; Koenderink, G. H.; Bakker, H. J. Molecular Origin of the Elastic State of Aqueous Hyaluronic Acid. J. Phys. Chem. B 2019, 123, 3043-3049.

(40) Ahmadi, M.; Hawke, L. G. D.; Goldansaz, H.; van Ruymbeke, E. Dynamics of Entangled Linear Supramolecular Chains with Sticky Side Groups: Influence of Hindered Fluctuations. Macromolecules 2015, 48, 7300-7310.

(41) Hackelbusch, S.; Rossow, T.; van Assenbergh, P.; Seiffert, S. Chain Dynamics in Supramolecular Polymer Networks. Macromolecules 2013, 46, 6273-6286.

(42) Cyphert, J. M.; Trempus, C. S.; Garantziotis, S. Size Matters: Molecular Weight Specificity of Hyaluronan Effects in Cell Biology. Int. J. Cell Biol. 2015, 2015, 563818.

(43) Maxa, J. J.; Chapados, C. Isotope effects in liquid water by infrared spectroscopy. III. $\mathrm{H} 2 \mathrm{O}$ and D2O spectra from 6000 to 0 $\mathrm{cm}^{-1}$. J. Chem. Phys. 2009, 131, 184505.

(44) Krezel, A.; Bal, W. A formula for correlating pKa values determined in $\mathrm{D} 2 \mathrm{O}$ and $\mathrm{H} 2 \mathrm{O}$. J. Inorg. Biochem. 2004, 98, 161-166.

(45) Lowry, K. M.; Beavers, E. M. Thermal stability of sodium hyaluronate in aqueous solution. J. Biomed. Mater. Res. 1994, 28, $1239-1244$

(46) Amunson, K. E.; Kubelka, J. On the temperature dependence of amide I frequencies of peptides in solution. J. Phys. Chem. B 2007, 111, 9993-9998.

(47) Selig, O.; Siffels, R.; Rezus, Y. L. Ultrasensitive Ultrafast Vibrational Spectroscopy Employing the Near Field of Gold Nanoantennas. Phys. Rev. Lett. 2015, 114, 233004.

(48) Blundell, C. D.; Deangelis, P. L.; Almond, A. Hyaluronan: the absence of amide-carboxylate hydrogen bonds and the chain conformation in aqueous solution are incompatible with stable secondary and tertiary structure models. Biochem. J. 2006, 396, 487498.

(49) Attili, S.; Borisov, O. V.; Richter, R. P. Films of end-grafted hyaluronan are a prototype of a brush of a strongly charged, semiflexible polyelectrolyte with intrinsic excluded volume. Biomacromolecules 2012, 13, 1466-1477.

(50) Giubertoni, G.; Pérez de Alba Ortíz, A.; Bano, F.; Zhang, X.; Linhardt, R. J.; Green, D. E.; DeAngelis, P. L.; Koenderink, G. H.; Richter, R. P.; Ensing, B.; Bakker, H. J. Strong reduction of the chain rigidity of hyaluronan by selective binding of $\mathrm{Ca}^{2+}$ ions. 2020, bioRxiv:2020.09.01.277194v1.

(51) Almond, A.; Deangelis, P. L.; Blundell, C. D. Hyaluronan: the local solution conformation determined by NMR and computer modeling is close to a contracted left-handed 4-fold helix. J. Mol. Biol. 2006, 358, 1256-1269.

(52) Almond, A.; Brass, A.; Sheehan, J. K. Oligosaccharides as Model Systems for Understanding Water-Biopolymer Interaction: Hydrated Dynamics of a Hyaluronan Decamer. J. Phys. Chem. B 2000, 104, 5634-5640.

(53) Dea, I. C. M.; Moorhouse, R.; Rees, D. A.; Arnott, S.; Guss, J. M.; Balazs, E. A. Hyaluronic acid: a novel, double helical molecule. Science 1973, 179, 560-562.

(54) Lodge, T. P.; Rotstein, N. A.; Prager, S. Dynamics of entangled polymer liquids. Do linear chains reptate?. Advances in Chemical Physics: Wiley, 1990; Vol. 79, pp 1-132.

(55) Dodero, A.; Williams, R.; Gagliardi, S.; Vicini, S.; Alloisio, M.; Castellano, M. A micro-rheological and rheological study of biopolymers solutions: Hyaluronic acid. Carbohydr. Polym. 2019, 203, 349-355.

(56) Oelschlaeger, C.; Cota Pinto Coelho, M.; Willenbacher, N. Chain flexibility and dynamics of polysaccharide hyaluronan in 
entangled solutions: a high frequency rheology and diffusing wave spectroscopy study. Biomacromolecules 2013, 14, 3689-3696.

(57) Dougherty, R. C. Temperature and pressure dependence of hydrogen bond strength: A perturbation molecular orbital approach. J. Chem. Phys. 1998, 109, 7372.

(58) Tanaka, F.; Edwards, S. F. Viscoelastic properties of physically crosslinked networks. J. Non-Newtonian Fluid Mech. 1992, 43, 273288.

(59) Ferry, J. D. Viscoelastic Properties of Polymers; John Wiley \& Sons: New York, 1980.

(60) Shamoun, R. F.; Hariri, H. H.; Ghostine, R. A.; Schlenoff, J. B. Thermal transformations in extruded saloplastic polyelectrolyte complexes. Macromolecules 2012, 45, 9759-9767.

(61) Spruijt, E.; Sprakel, J.; Lemmers, M.; Stuart, M. A.; van der Gucht, J. Relaxation dynamics at different time scales in electrostatic complexes: time-salt superposition. Phys. Rev. Lett. 2010, 105, 208301.

(62) Kovács, A.; Nyerges, B.; Izvekov, V. Vibrational analysis of Nacetyl-alpha-D-glucosamine and beta-D-glucuronic acid. J. Phys. Chem. B 2008, 112, 5728-5735.

(63) Hamm, P.; Zanni, M. Concepts and Methods of 2D Infrared Spectroscopy; Cambridge University Press: Cambridge, 2011; pp 636.

(64) Sheu, S.-Y.; Yang, D.-Y.; Selzle, H. L.; Schlag, E. W. Energetics of hydrogen bonds in peptides. Proc. Natl. Acad. Sci. U.S.A. 2003, 100, 12683-12687.

(65) Bolen, D. W.; Rose, G. D. Structure and energetics of the hydrogen-bonded backbone in protein folding. Annu. Rev. Biochem. 2008, 77, 339-362.

(66) Hamad, F. G.; Chen, Q.; Colby, R. H. Linear Viscoelasticity and Swelling of Polyelectrolyte Complex Coacervates. Macromolecules 2018, 51, 5547-5555.

(67) Reed, C. E.; Li, X.; Reed, W. F. The effects of $\mathrm{pH}$ on hyaluronate as observed by light scattering. Biopolymers 1989, 28, 1981-2000.

(68) Tarek, M.; Tobias, D. J. Role of protein-water hydrogen bond dynamics in the protein dynamical transition. Phys. Rev. Lett. 2002, $88,138101$.

(69) Giannotti, M. I.; Rinaudo, M.; Vancso, G. J. Force spectroscopy of hyaluronan by atomic force microscopy: from hydrogen-bonded networks toward single-chain behavior. Biomacromolecules 2007, 8, 2648-2652.

(70) Gerweck, L. E.; Seetharaman, K. Cellular $\mathrm{pH}$ gradient in tumor versus normal tissue: potential exploitation for the treatment of cancer. Cancer Res. 1996, 56, 1194-1198.

(71) Corbet, C.; Feron, O. Tumour acidosis: from the passenger to the driver's seat. Nat. Rev. Cancer 2017, 17, 577-593.

(72) Erra Díaz, F.; Ochoa, V.; Merlotti, A.; Dantas, E.; Mazzitelli, I.; Gonzalez Polo, V.; Sabatté, J.; Amigorena, S.; Segura, E.; Geffner, J. Extracellular Acidosis and mTOR Inhibition Drive the Differentiation of Human Monocyte-Derived Dendritic Cells. Cell Rep. 2020, 31, 107613.

(73) Erra Díaz, F.; Dantas, E.; Geffner, J. Unravelling the Interplay between Extracellular Acidosis and Immune Cells. Mediators Inflammation 2018, 2018, 1218297.

(74) Schmaljohann, D. Thermo- and $\mathrm{pH}$-responsive polymers in drug delivery 方. Adv. Drug Delivery Rev. 2006, 58, 1655-1670.

(75) Singh, A.; Corvelli, M.; Unterman, S. A.; Wepasnick, K. A.; McDonnell, P.; Elisseeff, J. H. Enhanced lubrication on tissue and biomaterial surfaces through peptide-mediated binding of hyaluronic acid. Nat. Mater. 2014, 13, 988-995.

(76) Meng, F.; Pritchard, R. H.; Terentjev, E. M. Stress Relaxation, Dynamics, and Plasticity of Transient Polymer Networks. Macromolecules 2016, 49, 2843-2852. 